\title{
Análise das práticas de planejamento e controle da produção em fornecedores da cadeia automotiva brasileira
}

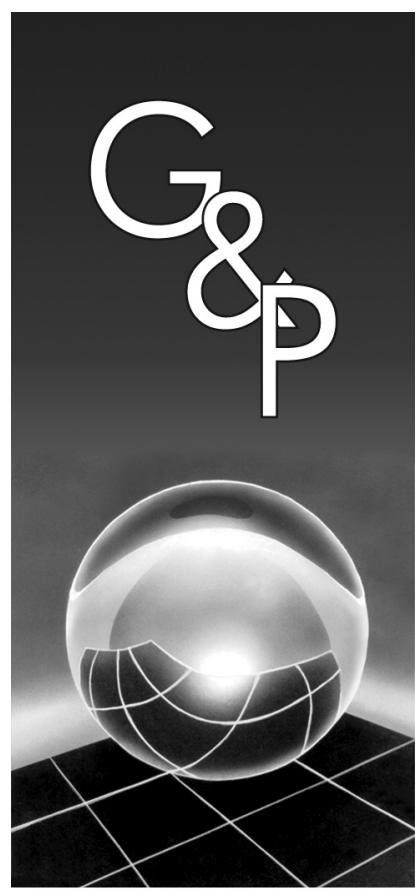

\author{
Marco Aurélio de Mesquita \\ Roberto Lopes de Castro
}

\begin{abstract}
Resumo
As empresas do segmento automotivo estão inseridas em um mercado bastante competitivo e sujeito às oscilações da economia. As montadoras, agentes de maior poder na cadeia de suprimentos, aderiram às práticas just-in-time e, posteriormente, ao lean production, visando aumento da eficiência e redução de estoques. Este trabalho apresenta uma análise das práticas de planejamento e controle da produção dos fornecedores da cadeia automotiva brasileira. Neste propósito, realizou-se um survey junto aos fornecedores de primeira e segunda camada, utilizando-se como instrumento de coleta de dados um questionário auto-aplicado. Dentre os resultados obtidos, destacam-se: (i) ausência de diferenças significativas entre fornecedores nível um e dois; (ii) necessidade de aprimorar a coordenação cliente-fornecedor; (iii) busca de "ferramentas" mais eficientes para programação da produção ("programação finita"); (iv) grande lacuna entre teoria e prática em programação da produção, atividade crítica para o desempenho dos fornecedores.
\end{abstract}

Palavras-chave: Planejamento e controle da produção. Controle de estoques. Cadeia automotiva.

\section{Introdução}

A cadeia automotiva brasileira tem sido objeto de várias pesquisas em gestão de operações devido ao seu rápido crescimento e à sua grande importância para a economia do país. Segundo dados da Associação Nacional dos Fabricantes de Veículos Automotores - ANFAVEA, a produção de veículos e as exportações bateram recorde no Brasil em 2006, atingindo 2,61 milhões e 842,8 mil unidades, respectivamente (ANFAVEA, 2007). Estes números colocam o país entre os grandes produtores mundiais de veículos, conforme apresentado na Tabela 1.

Salerno et al. (2003) apontam que a abertura de mercado e a globalização, que ocorreram nos anos 90 , associadas à promessa de crescimento de países emergentes, como Brasil, Argentina, México, China e Índia, possibilitaram a entrada de novas montadoras no Brasil. Aliado a isso, a busca pela adequação aos princípios do Sistema Toyota de produção nos anos 80 e 90, incluindo suprimento just-in-time (JIT), trabalho em grupo, programas de qualidade total e automação, resultou em intensa reestruturação das empresas, modificando as relações entre as montadoras e seus fornecedores.

A indústria brasileira de autopeças também passou por grandes transformações nos anos 90, devido à nova estratégia de operações das montadoras em relação à cadeia de suprimentos e às políticas governamentais para o setor automotivo. Conceição (2001) aponta que o setor passou por uma "modernização desarticulada", com reflexos diferentes para montadoras e fabricantes de autopeças. As montadoras modernizaram as unidades industriais e expandiram a produção em resposta ao crescimento do mercado consumidor. Por outro lado, o setor de autopeças passou por uma profunda crise estrutural, com fechamento de fábricas e aumento da importação de produtos acabados. Com a retomada do crescimento da produção, aumentou a pressão por fornecimento com prazos curtos, maior qualidade e menores custos. Esta pressão estende-se também para os fornecedores de segunda e terceira camadas da cadeia de suprimentos, que atendem um número maior de clientes e com maior variedade de produtos.

Posthuma (1995) realizou uma pesquisa para identificar os desdobramentos da adoção da lógica JIT pelas montadoras nas práticas de reposição de estoques e planejamento da produção dos fornecedores de autopeças. Verificou-se que a falta de uma programação firme de médio prazo dificultava o planejamento da produção e das entregas, inviabilizando a adoção do JIT. 
Tabela 1. Produção mundial de veículos. Fonte: Anfavea (2007).

\begin{tabular}{clc}
\hline Ordem & \multicolumn{1}{c}{ País } & Produção (milhões) em 2006 \\
\hline 1 & Japão & 11,484 \\
2 & Estados Unidos & 11,264 \\
3 & China & 7,189 \\
4 & Alemanha & 5,820 \\
5 & Coréia do Sul & 3,936 \\
6 & França & 3,169 \\
7 & Espanha & 2,777 \\
8 & Brasil & 2,611 \\
9 & Canadá & 2,572 \\
10 & México & 2,046 \\
11 & Índia & 1,944 \\
12 & Reino Unido & 1,648 \\
\hline
\end{tabular}

Arkader (2001) revela uma evolução no relacionamento cliente-fornecedor, concentrada em seus aspectos mais operacionais. No entanto, da perspectiva dos fornecedores, há ainda uma grande distância a ser percorrida para a implantação efetiva do suprimento enxuto. Neste sentido, o estudo revela que um avanço mais significativo esbarra na falta de visão estratégica das montadoras com relação à gestão de suas cadeias de suprimentos.

Salerno et al. (2003) apontam a existência de um maior distanciamento das "melhores práticas" à montante da cadeia automotiva brasileira. Estes fornecedores sofrem dupla pressão: por um lado, a pressão dos clientes (nível um na cadeia) por prazo, custo e qualidade e, por outro lado, a pressão dos fornecedores oligopolistas de matériaprima, que impõem condições comerciais de fornecimento, como prazo de entrega, lotes mínimos e preços.

Oproblema desta pesquisa origina-se no aparente desempenho insatisfatório das áreas de planejamento e controle da produção dos fornecedores de nível dois da indústria automotiva brasileira, caracterizado por atrasos frequientes nas entregas e altos custos de estoques. Este trabalho apresenta uma análise das práticas de planejamento e controle da produção dos fornecedores da cadeia automotiva brasileira. Para isto, realizou-se um levantamento bibliográfico dos principais modelos de $\mathrm{PCP}$ e, a seguir, uma pesquisa do tipo survey junto aos fornecedores de primeira e segunda camada, utilizando-se como instrumento de coleta de dados um questionário auto-aplicado.

Este artigo está dividido em cinco seções. A seção 2 apresenta uma revisão dos conceitos de gestão da cadeia de suprimentos e de planejamento e controle da produção. Estes conceitos, bastante difundidos no meio acadêmico, serão confrontados com as práticas levantadas na pesquisa de campo. As questões e o método de pesquisa utilizados são detalhados na seção 3. A seção 4 apresenta e discute os resultados obtidos na coleta de dados. Por fim, a seção 5 encerra o artigo com as conclusões da pesquisa.

\section{Referencial teórico}

\subsection{Gestão da cadeia de suprimentos}

A gestão da cadeia de suprimentos compreende o planejamento e o controle de todas as operações incluídas nas atividades de suprimento e compras, transformação e todas as atividades logísticas de distribuição física dos produtos. A gestão da cadeia de suprimentos é uma função integradora com propósito principal de conectar as áreas e processos comerciais das empresas da cadeia, buscando um modelo de negócios coeso e eficiente (CSCMP, 2006).

Chopra e Meindl (2001) destacam que a competição ocorrerá cada vez mais entre cadeias produtivas, e não mais entre empresas isoladas. Como consequiência, clientes e fornecedores precisam adotar estratégias voltadas para a formação de parcerias e troca de informações, de modo a crescerem e se beneficiarem mutuamente. Em outras palavras, quando a ponta da corrente "puxa", todos os elos se beneficiam e crescem, desde que estejam alinhados e tenham políticas e estratégias bem definidas de parceria e colaboração. Esta constatação empírica é formalmente comprovada em Frohlich e Westbrook (2001) que, com base em dados de uma pesquisa internacional com 322 empresas do setor metal-mecânico, demonstram a existência de uma correlação positiva entre o grau de integração e o desempenho da cadeia de suprimentos.

Um dos fenômenos mais discutidos em gestão da cadeia de suprimentos, tanto no meio acadêmico quanto empresarial, tem sido o denominado "efeito chicote" (bullwhip effect), que consiste na amplificação da variação da demanda a montante das cadeias de suprimentos. Esta amplificação dificulta a gestão de estoques e o gerenciamento de pedidos na cadeia (LEE et al., 2004). Há diversos fatores que contribuem para a ocorrência do efeito chicote em uma cadeia de suprimentos, com destaque para a política de reposição de estoques e formação de lotes, as dificuldades de previsão de demanda e as estratégias comerciais de vendas.

$\mathrm{Na}$ indústria, várias iniciativas como, por exemplo, Vendor Managed Inventory - VMI e Collaborative Planning, Forecasting and Replenishment - CPFR, abordam o problema, evidenciando a decorrência nociva do efeito chicote sobre a eficiência das cadeias de suprimento. As empresas buscam reduzir as incertezas quanto à demanda na cadeia para reduzir o efeito chicote, mudando o padrão de reposição de estoques, além de investir em ferramentas de tecnologia da informação (LEE et al., 2004). A importância do compartilhamento de informações na gestão eficiente das cadeias de supri- 
mentos pode ser atestada pela grande quantidade de trabalhos sobre o tema (HUANG et al., 2003).

$\mathrm{Na}$ indústria automotiva, são as empresas montadoras que "puxam" os outros elos da cadeia. De acordo com Womack et al. (1992), as montadoras podem ser submetidas a mudanças repentinas no mix de produtos demandados pelos consumidores, conforme flutuações do mercado automobilístico. Estas mudanças seriam inevitáveis e imprevisíveis, provocando cancelamento repentino de pedidos ou inserção de pedidos urgentes junto aos fornecedores. Estas práticas contrariam os preceitos de Gestão da Cadeia de Suprimentos e provocam a ocorrência do efeito chicote na cadeia.

Atualmente, as empresas da cadeia automotiva buscam superar as dificuldades de gestão da cadeia pela adoção do modelo "Lean Manufacturing" (WOMACK; JONES, 1996). Este modelo, que tem origem no Sistema Toyota de Produção - STP, consolidou as práticas de produção just-in-time (produção puxada e pequenos lotes) com a redução do número de fornecedores e exigência de cumprimento de prazos e qualidade assegurada. Hines et al. (2004) apresentam a evolução do conceito de produção enxuta, cuja abrangência estendeu-se do chão de fábrica para a cadeia de suprimentos, e o foco em redução de custos deu lugar à ênfase no valor agregado ao cliente.

Cagliano et al. (2006) demonstram, a partir de um survey com 297 empresas européias de manufatura, que a adoção do modelo de produção enxuta contribui significativamente para a integração dos fluxos de materiais (físico) e informação, com impacto positivo no resultado operacional das empresas. Quayle (2003), Wu (2003), Olhager e Selldin (2004), Fynes et al. (2005) e Szwejczewski et al. (2005) também abordam as práticas de gestão da cadeia de suprimentos e manufatura em países europeus e nos Estados Unidos.

Lambert e Cooper (2000) destacam a importância da melhoria e integração dos processos internos para a efetiva integração à cadeia de suprimentos. Estes processos incluem: (i) gestão de relacionamentos e atendimento ao cliente, (ii) gestão da demanda, (iii) gerenciamento de pedidos, (iv) gerência da produção e suprimentos; (v) desenvolvimento de produtos e (vi) logística reversa. Neste contexto, destaca-se a importância da adequação do sistema de planejamento e controle da produção e da estrutura organizacional da empresa de manufatura para a sua efetiva integração na cadeia de suprimentos. A seção 2.2, a seguir, aborda os modelos de planejamento e controle da produção, que são o foco desta pesquisa.

\subsection{Planejamento e controle da produção}

Devido à complexidade do setor automotivo e à necessidade imperativa de integração dos fornecedores na cadeia de suprimentos, torna-se imprescindível a disponibilidade de um sistema de planejamento e controle da produção eficaz, que permita à empresa gerenciar a demanda, os materiais, a capacidade produtiva e a produção. A eficácia do PCP pode ser avaliada pelo alcance dos objetivos de redução dos lead times de produção, dos custos de estoque (matéria-prima, materiais em processo e produtos acabados) e de produção (ociosidade, horas extras, etc), cumprimento de prazos e agilidade de resposta diante de alterações de demanda.

Nesta seção, apresentam-se alguns dos modelos mais importantes de PCP, incluindo: planejamento hierárquico da Produção (PHP), manufacturing resources planning (MRPII), just-in-time - kanban (JIT), teoria das restrições (TOC - theory of constrains) e constant work-in process (CONWIP). Para uma revisão mais completa, recomenda-se consultar Stevenson et al. (2005) e Vollmann et al. (1997).

\subsubsection{Planejamento hierárquico da produção (PHP)}

No modelo planejamento hierárquico da produção, originalmente proposto por Hax e Meal (1975) e Bitran et al. (1981, 1982), o problema de planejamento da produção é dividido em "subproblemas", conforme o nível hierárquico de decisão. Estes "subproblemas" são formulados matematicamente a partir de modelos de otimização e devem ser resolvidos seqüencialmente, do nível estratégico ao nível operacional. As soluções de nível superior na hierarquia constituem restrições para as decisões dos níveis abaixo, enquanto os resultados dos níveis inferiores realimentam os níveis superiores de decisão (HAX; CANDEA, 1984).

Tipicamente, os modelos PHP apresentam dois níveis hierárquicos: planejamento agregado e planejamento detalhado. Inicia-se pela solução do modelo de planejamento agregado, onde os dados de demanda e recursos são considerados de forma agregada (famílias de produtos, centros de produção e período mensal). Este plano agregado é detalhado a partir de um modelo de desagregação, resultando no programa mestre de produção. A execução do programa mestre de produção requer ainda um nível de decisão mais operacional, que usualmente não está incluído no modelo PHP e que consiste na programação detalhada da produção (STOOP; WIERS, 1996; GUPTA, 2002; STEVENSON et al., 2005).

Apesar de sua estrutura lógica, o modelo PHP raramente é encontrado nas empresas. Dentre as razões que explicam este fato, destaca-se, por um lado a necessidade de grandes simplificações na modelagem e, por outro, a elevada complexidade dos métodos de solução, o que torna estes modelos de difícil compreensão e baixa aceitação pelos profissionais de PCP (MESQUITA; SANTORO, 2004).

Buxey (2005) destaca que o problema de planejamento agregado torna-se relevante apenas para empresas 
com produção para estoque (MTS - make to stock) e demanda altamente sazonal. Ainda assim, as empresas deste segmento optam por estratégias de produção predeterminadas, em detrimento do uso de modelos para tomada de decisão. Nos fornecedores da cadeia automotiva, com produção contra pedido (MTO - make to order), as decisões operacionais de programação e controle da produção (scheduling) assumem maior importância em relação às decisões de nível tático, tratadas nos modelos de planejamento agregado da produção (STEVENSON et al., 2005).

\subsubsection{Manufacturing resources planning - MRPII}

O modelo MRPII surgiu nos EUA, na década de 70, com a denominação inicial de material requirements planning - MRP. Antes do surgimento do MRP, a maioria dos sistemas de controle de materiais e estoques na produção baseava-se em alguma variação do modelo de reposição de estoques pelo ponto de pedido (PLOSSL, 1994). O MRP quebra este paradigma, quando propõe que as demandas de matérias-primas e componentes sejam tratadas como demandas "dependentes", ou seja, a administração de materiais deve considerar a correlação direta entre a demanda destes materiais e a demanda de produtos acabados.

O modelo MRP é caracterizado como um sistema de produção empurrada, que gera as ordens de produção e compras de materiais conforme o programa mestre de produção, as listas de materiais dos produtos e os níveis de estoques. A partir dos tempos de resposta (lead times) dos processos, determinam-se os instantes em que as ordens devem ser liberadas, aplicando uma lógica de programação para trás. Um maior detalhamento sobre o método de cálculo das necessidades de materiais pode ser obtido em Hopp e Spearman (2000).

Para verificar a viabilidade do programa gerado pelo MRP, deve-se proceder ao carregamento dos centros de produção, isto é, calcular as cargas de trabalho (normalmente em minutos ou horas) em cada centro, referentes ao conjunto de ordens de produção gerado, e confrontar estas cargas com as respectivas capacidades. Havendo divergências, deve-se tentar expandir a capacidade (por exemplo, usando-se horas extras) ou alterar a programação. Este procedimento iterativo de ajuste foi denominado MRP de ciclo fechado (closedloop MRP).

Na década de 80, o modelo MRP evolui para o MRPII (manufacturing resource planning), incorporando novas funcionalidades. No início da década de 90, surgiram os sistemas integrados de gestão empresarial denominados ERP (enterprise resource planning). Esta nova geração, além de incluir o módulo industrial baseado na lógica MRPII, inclui vários outros aspectos da gestão empresarial como, por exemplo, os módulos contábil, financeiro, comercial, recursos humanos, engenharia, etc (LAURINDO; MESQUITA, 2000).

O modelo MRPII apresenta uma estrutura hierárquica similar ao PHP e tornou-se um padrão importante de Sistema de PCP (VOLLMANN et al., 1997). Hopp e Spearman (2000) relacionam algumas das limitações deste modelo, dentre as quais se destacam: (i) a hipótese implícita de capacidade infinita, que usualmente gera programas de produção imperfeitos e dificuldades de estimação dos tempos de resposta (lead times), (ii) a ausência de um mecanismo eficaz de controle da produção ("produção empurrada").

Stevenson et al. (2005) concluem que apesar da grande difusão do modelo MRPII, este não é adequado às necessidades de fornecedores em produção MTO, devido às dificuldades de configuração e operação do sistema. Por outro lado, a popularização dos ERP's como sistemas de informação corporativos pode proporcionar aos pequenos fornecedores a desejada integração interna e externa na cadeia de suprimentos.

\subsubsection{Just-in-time - kanban}

O mecanismo kanban de produção puxada, com o nivelamento da produção, constitui um dos fundamentos do sistema just-in-time (JIT). Para implantá-lo no chão de fábrica, Ohno recorreu à analogia com o funcionamento de um supermercado. Segundo o autor:

"From the supermarket we got the idea of viewing the earlier process in a production line as a kind of store. The later process (customer) goes to earlier process (supermarket) to acquire the needed parts (commodities) at the time and in the quantity needed. The earlier process immediately produces the quantity just taken (re-stocking the shelves)." (OHNO, 1988, p.26)

A sinalização do consumo e a autorização para reposição do estoque são feitas pelo uso de cartões (kanbans), que são destacados dos containers retirados e fixados no painel do operador responsável pela reposição. Há duas formas principais de implantação da lógica kanban, com um cartão (produção) ou dois cartões (produção e transporte).

Incluindo outras iniciativas de melhoria (redução de setups, zero defeitos, arranjo celular, etc), o modelo JIT produziu aumentos significativos de produtividade na indústria automobilística japonesa, tornando-se padrão de produção de automóveis em diferentes países ("produção puxada"). Descrições mais detalhadas do tradicional modelo JIT-kanban são encontradas em Schonberger (1983) e Karmarkar (1989).

Os conceitos do modelo just-in-time foram incorporados pelos fabricantes norte-americanos, europeus e de 
países emergentes como o Brasil. Este modelo evoluiu, dando origem ao "sistema de produção enxuta" ou "lean manufacturing" (WOMACK et al., 1992; WOMACK; JONES, 1996).

Stevenson et al. (2005) e Hopp e Spearman (2000) questionam a adequação do modelo JIT-kanban em sistemas de produção MTO. Neste trabalho, pretende-se analisar os desdobramentos da adoção deste modelo, concebido para sistemas de produção em massa pelas montadoras sobre os fornecedores com produção intermitente.

\subsubsection{Constant work-in-process - CONWIP}

Spearman et al. (1989) apresentam o modelo CONWIP como parte de um sistema hierárquico de planejamento e controle da produção. Determinadas as metas de produção, a liberação das ordens deve ser feita, mantendo constante a carga de trabalho (estoque em processo) na fábrica. No CONWIP, conforme representado na Figura 1, o controle de estoque em processo é feito na linha como um todo; os cartões não especificam o produto, mas sim a carga de trabalho. Estas diferenças permitem a implantação do modelo em sistema MTO, preservando a característica de produção puxada (HOPP; SPEARMAN, 2004).

Hopp e Spearman (2000) desenvolvem, a partir do clássico PHP, uma estrutura de sistema de planejamento e controle da produção, cuja base operacional (programação detalhada e controle da produção) apóia-se no modelo CONWIP. Framinan et al. (2003) apresentam uma completa revisão bibliográfica sobre o modelo CONWIP.

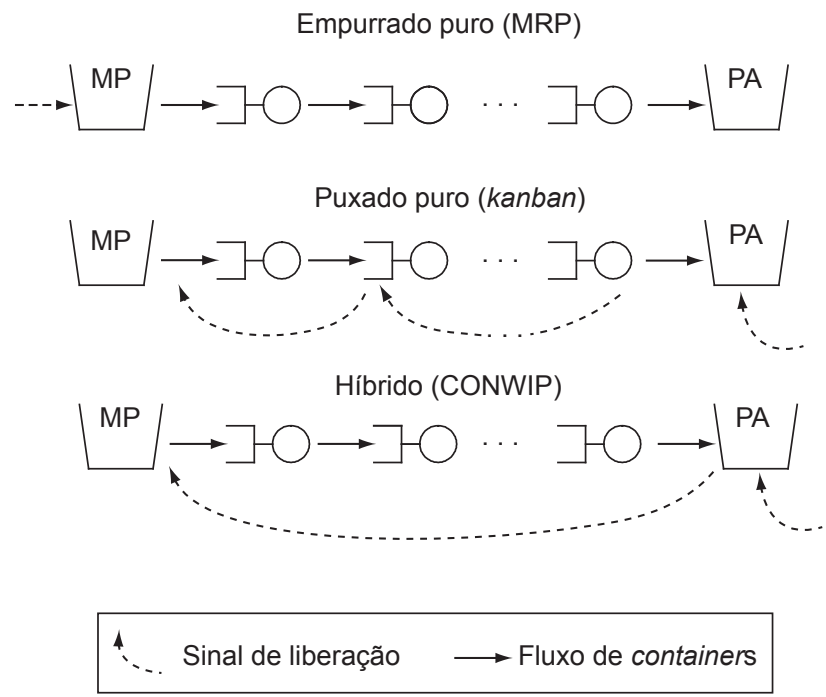

Figura 1. Mecanismos de produção empurrada e puxada. Adaptado de Hopp e Spearman (2000).

\subsubsection{Teoria das restrições (theory of constraints - TOC)}

Esta abordagem tem origem no modelo optimized production technology (OPT), proposto por Eliyahu M. Goldratt e que consiste em um algoritmo de programação finita da produção orientado para a maximização do fluxo no recurso crítico ou gargalo. Este conceito foi expandido, dando origem à "Teoria das Restrições", que inclui uma filosofia de melhoria contínua, uma proposta de sistema de avaliação de desempenho e um módulo de programação e controle da produção baseado no recurso gargalo (SPENCER; COX, 1995).

A teoria das restrições aponta três indicadores fundamentais de desempenho: vazão, estoques e despesas operacionais. A vazão do sistema de produção está diretamente associada à velocidade dos ganhos para a empresa (lucro e retorno sobre investimentos). Os estoques devem ser gerenciados de forma a garantir o fluxo máximo (ganhos), funcionando como pulmões para incertezas do mercado e dos processos de produção nos recursos críticos. As despesas operacionais são todos os desembolsos necessários para transformar os estoques em vazão (ganhos).

$\mathrm{Na}$ TOC, o processo de melhoria contínua é constituído por cinco passos, a saber:

a) identificar o recurso crítico do sistema;

b) explorar o potencial deste recurso;

c) subordinar os demais recursos ao recurso crítico;

d) relaxar a restrição que limita o potencial do recurso crítico; e

e) retornar ao passo a, identificando novas oportunidades de melhoria.

No ambiente da produção, os passos b, c e d estão diretamente associados à programação e controle da produção, evitando que o recurso crítico fique ocioso por falta de material, por tempos longos de preparação (setup), por problemas de qualidade e manutenção, etc.

O método de programação e controle, denominado drumbuffer-rope (DBR), consiste basicamente em: (i) programar o recurso crítico ou gargalo - tambor; (ii) dimensionar estoque intermediário para proteção do recurso crítico pulmão; (iii) puxar a produção a partir do recurso gargalo - corda. Desta forma, o modelo DBR caracteriza-se pelo uso de técnicas de programação finita e um mecanismo de produção puxada. Em Blackstone (2001), o leitor interessado encontrará um detalhamento do método DBR.

Uma comparação entre os modelos DBR e MRP é apresentada por Steele et al. (2005). Jodlbauer e Huber (2007) apresentam resultados de um estudo de simulação em que são comparados os métodos de PCP apresentados nesta seção (MRP, kanban, CONWIP e DBR). Por fim, destaca-se também que os modelos acima apresentados podem ser combinados na prática, dando origem aos sistemas híbridos e proporcionando às empresas sistemas de PCP mais adaptados às suas necessidades. 


\section{Delineamento da pesquisa}

Esta pesquisa tem como objeto de estudo os fornecedores de autopeças da indústria automotiva brasileira. O objetivo é avaliar o desempenho das áreas de planejamento e controle da produção dos fornecedores de nível dois, confrontando suas práticas e seus resultados com os de fornecedores da primeira camada.

Uma justificativa para este estudo é que poucas pesquisas são voltadas para os fornecedores de segunda e terceira camadas, como salientam Miranda (2000) e Salerno et al. (2003). Em adição, não foram encontrados na literatura estudos específicos a respeito da integração interna e das práticas de PCP nos fornecedores, que conforme discutido na seção anterior, são dois aspectos críticos para a efetiva integração destes na cadeia de suprimentos (LAMBERT; COOPER, 2000). Assim, para orientar a condução deste estudo, são formuladas duas questões de pesquisa:

Questão 1: Os fornecedores do nível 2 estão integrados na cadeia de suprimentos?

Questão 2: Os modelos e práticas de PCP no nível 2 são adequados?

O método de pesquisa adotado neste trabalho é o survey, que tem por objetivo a descrição de fenômenos através da coleta de estruturada de dados, com a utilização de questionários ou entrevistas (BRYMAN, 1989). É um método que permite a participação de um número grande de entidades, sem a intervenção direta do pesquisador na coleta de dados.

Forza (2002) descreve três tipos básicos de pesquisas baseadas em survey: exploratória, descritiva e comprobatória ou "theory testing". Nesta divisão, a presente pesquisa pode ser classificada como descritiva-exploratória, tendo como objetivo o levantamento e análise das práticas e desempenho do PCP nas empresas consideradas.

Para efeito deste survey, as empresas que compõem o universo amostral são aquelas associadas ao Sindipeças (461 empresas, em 2004). Devido à limitação de recursos e tempo, optou-se pela redução desta população às empresas que participaram do "censo automotivo brasileiro", realizado por Salerno et al. (2003).

Nesta pesquisa, foram excluídos os grandes fornecedores de matéria-prima na base da cadeia (fabricantes de vidro, pneus, tintas, aço e plásticos em geral), fabricantes de materiais auxiliares (borracha, combustíveis e lubrificantes) e empresas de serviços, pois apresentam características muito diversas das demais empresas da cadeia automotiva.

O questionário utilizado é dividido em cinco partes: (i) identificação da empresa; (ii) previsão de demanda; (iii) programação da produção; (iv) estoques e (v) avaliação do PCP. Uma primeira versão deste questionário foi enviada a três fornecedores para avaliar a compreensão das questões e eliminar dúvidas de interpretação. A partir deste pré-teste, procedeu-se uma revisão do questionário, cuja versão final encontra-se em Castro (2005).

Elaborado e testado o questionário, iniciaram-se os contatos telefônicos e via e-mail, buscando alcançar os profissionais das empresas mais indicados para participação na pesquisa. Foram enviados 220 questionários, tendo sido obtido um retorno de $21 \%$ (46 respostas). Segundo dados divulgados pelo Sindipeças (2005), o tamanho da amostra representa cerca de $10 \%$ das empresas filiadas à entidade. A Figura 2 destaca a variedade de produtos do segmento automobilístico produzidos pelas empresas participantes da amostra, conforme classificação do Sindipeças.

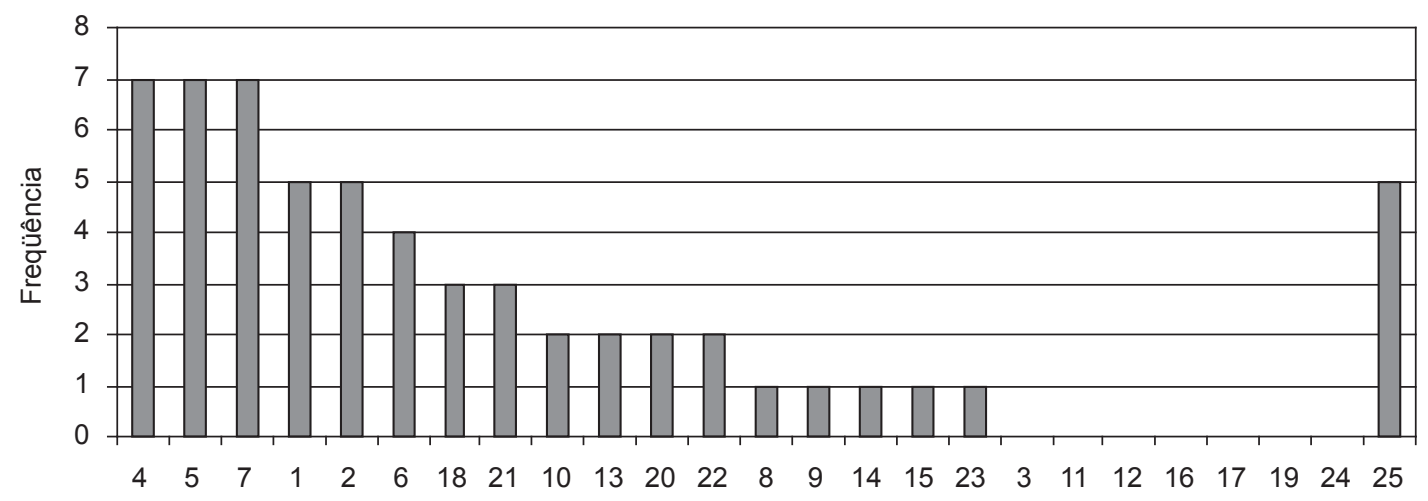

\begin{tabular}{|c|c|c|c|c|}
\hline 1. Estampados & 6. Peças plásticas & 11. Carburação e injeção & 16. Eletromecânicos & 21. Transmissão/Engr. \\
\hline 2. Motores e suas partes & 7. Artefatos de borracha & 12. Rolamentos & 17. Material de fricção & 22. Escapamentos \\
\hline 3. Material elétrico & 8. Molas mecânicas & 13. Ferragens & 18. Suspensão e direção & 23. Embreagem \\
\hline 4. Usinados & 9. Cabos de comando & 14. Peças sinterizadas & 19. Assentos e revest & 24. Instrumentação \\
\hline 5. Fundidos e forjados & 10. Rodas & 15. Parafusos e porcas & 20. Peças de acabamento & 25. Outros \\
\hline
\end{tabular}

Figura 2. Distribuição por família de produtos. 
A Figura 3 fornece a distribuição das empresas respondentes no que se refere à posição predominante declarada na cadeia de suprimentos. Para efeito específico da comparação entre os níveis de fornecimento na cadeia automotiva, os fornecedores níveis 2 e 3 (que juntos somam $28 \%$ da amostra) foram agrupados, e as empresas montadoras e voltadas principalmente para exportação e mercado de reposição foram desconsideradas.

\section{Análise dos resultados}

Nesta seção, são apresentados e discutidos os principais resultados do survey, com ênfase na comparação entre fornecedores de primeira e de segunda camada (Tabela 2). Uma análise completa dos resultados da presente pesquisa está disponível em Castro (2005).

O uso sistemático de previsão de demanda é mais comum no elo mais próximo à montadora, mas, em geral, os motivos para sua adoção estão direcionados ao planejamento da capacidade de produção e às decisões de aquisição antecipada de materiais junto aos fornecedores. As técnicas usadas para previsão de demanda são caracterizadas pela simplicidade (média móvel e opinião de especialistas), produzindo resultados satisfatórios para as aplicações a que se destinam, conforme avaliação dos participantes.

Quanto à utilização de técnicas de planejamento da produção, verificou-se que não há uniformidade entre as empresas pesquisadas, prevalecendo o MRP II como a lógica mais citada, seguido do OPT. Vale ressaltar, que apesar da grande difusão dos modelos lean manufacturing e JIT na cadeia automotiva, poucos fornecedores citaram a adoção do kanban, o que reforça o argumento de Stevenson et al. (2005) sobre a inadequação deste modelo para fornecedores MTO.

O MRP II tem forte presença nas empresas, mas sua aplicação fica restrita à administração de materiais (MRP I). Os entrevistados não citaram o uso de modelos de programação finita (scheduling), o que pode constituir-se numa oportunidade de melhoria, uma vez que a programação da produção foi o item reportado como mais importante dentre as atividades de PCP.

Tabela 2. Síntese dos resultados.

\begin{tabular}{lcc}
\hline \multicolumn{1}{c}{ Tópico } & Nível 1 & Nível 2 \\
\hline $\begin{array}{l}\text { Faz previsão de demanda } \\
\text { sistematicamente? }\end{array}$ & $96 \%$ & $54 \%$ \\
\hline $\begin{array}{l}\text { Modelo de planejamento da } \\
\text { produção }\end{array}$ & \\
MRPII & $68 \%$ & $60 \%$ \\
OPT & $26 \%$ & $30 \%$ \\
JIT & $4 \%$ & $10 \%$ \\
\hline Modo de produção & & \\
MTO (make to order) & $26 \%$ & $77 \%$ \\
MTS (make to stock) & $9 \%$ & $0 \%$ \\
MTO e MTS & $39 \%$ & $23 \%$ \\
ATO (assembly to order) & $13 \%$ & $0 \%$ \\
Produção contínua & $13 \%$ & $0 \%$ \\
\hline
\end{tabular}

Grau de colaboração (escala de

0 a 5)

$\begin{array}{lll}\text { Com clientes } & 3,7 & 4,0 \\ \text { Com fornecedores } & 3,7 & 3,8\end{array}$

Cobertura de estoque

Matéria-prima 15 dias 15 dias

Produto acabado 5 dias 8 dias

\begin{tabular}{|c|c|c|}
\hline \multicolumn{3}{|l|}{$\begin{array}{l}\text { Acurácia dos registros de estoques } \\
\text { ("acima de 98\%") }\end{array}$} \\
\hline Matéria-prima & $26 \%$ & $23 \%$ \\
\hline Produto acabado & $35 \%$ & $31 \%$ \\
\hline $\begin{array}{l}\text { Nível de estoque (respostas } \\
\text { "alto"): }\end{array}$ & $17 \%$ & $23 \%$ \\
\hline Entregas no prazo (mediana) (\%) & 95 & 95 \\
\hline $\begin{array}{l}\text { Avaliação do PCP (escala de } \\
0 \text { a 5) }\end{array}$ & 3,8 & 3,5 \\
\hline $\begin{array}{l}\text { Atividades de PCP mais } \\
\text { importantes }\end{array}$ & $\begin{array}{l}\text { - Program } \\
\text { produção; } \\
\text { - Previsão }\end{array}$ & \\
\hline $\begin{array}{l}\text { Atividades de PCP menos } \\
\text { importantes }\end{array}$ & $\begin{array}{l}\text { - Controle } \\
\text { - Controle }\end{array}$ & $\begin{array}{l}\text { ição; e } \\
\text { ues. }\end{array}$ \\
\hline Principais dificuldades do PCP & $\begin{array}{l}\text { - Alteraçã } \\
\text { pedidos; } \\
\text { - Baixa co } \\
\text { entrega de } \\
\text { - Sistema } \\
\text { deficiente; } \\
\text { - Baixa pr } \\
\text { tros de est }\end{array}$ & $\begin{array}{l}\text { te de } \\
\text { ade de } \\
\text { hação } \\
\text { s regis- }\end{array}$ \\
\hline
\end{tabular}

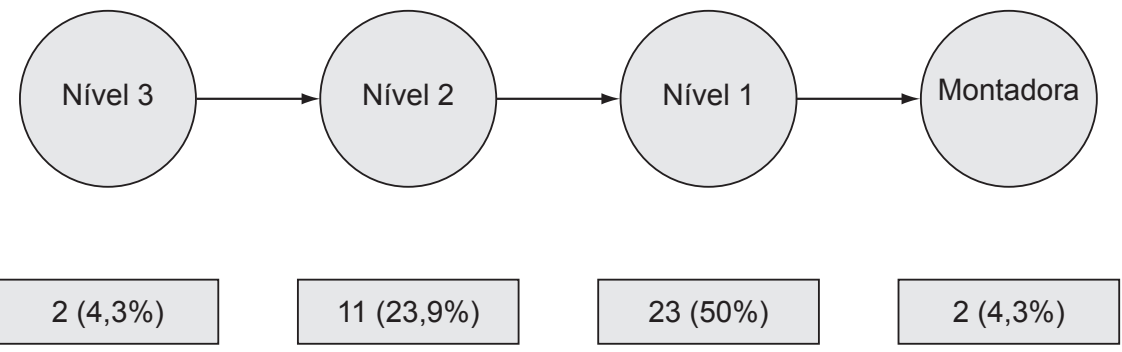

Mercado de reposição ou exportação

$8(17,4 \%)$

Figura 3. Distribuição da posição predominante na cadeia. 
Estes modelos podem ser desenvolvidos sob medida ou então pela aquisição de softwares de mercado, garantindo a integração do módulo de programação detalhada com o Sistema ERP da empresa. O controle da produção também pode ser melhorado, a partir da adoção da produção puxada (CONWIP ou OPT), aspecto também pouco mencionado nas respostas.

A maioria dos respondentes julga ter um bom nível de informação para o PCP e níveis de estoque adequados, ainda que tenham declarado carregar cerca de quinze dias de estoque de matéria-prima e cinco dias de estoque de produto acabado (valores medianos). Os números são elevados para os padrões da produção enxuta e confirmam resultados de outras pesquisas na área automobilística, ou seja, que o JIT se traduz na transferência de estoque para montante da cadeia (MIRANDA, 2000) e que é justamente este estoque que proporciona ao fornecedor flexibilidade no atendimento da demanda (ROLDAN, 2003). Verificou-se, nesta pesquisa, que o estoque de produto acabado é ligeiramente maior no nível 2 do que no nível 1.

Cerca de $70 \%$ dos respondentes afirmaram que o maior problema enfrentado pelo PCP é a alteração freqüente da programação pelo cliente e a alta incidência de pedidos urgentes. A falta de coordenação nos elos da cadeia é contornada com o aumento dos estoques de segurança e reservas de capacidade.

Confrontando-se os resultados desta pesquisa com Miranda (2000), pode-se concluir que houve um aumento significativo na troca eletrônica de dados na cadeia, pela utilização de recursos como a Internet e o EDI. No entanto, esta prática está mais difundida no nível 1 da cadeia de suprimentos. No que diz respeito ao planejamento colaborativo, as barreiras na sua difusão parecem estar mais relacionadas aos aspectos culturais e não tecnológicos, sendo este um aspecto que pode ser aprofundado em pesquisas futuras.

Há uma ampla variedade de softwares utilizados pelas empresas respondentes na programação da produção, tendo sido identificados 20 tipos diferentes de softwares em 46 respostas. Esta diversidade dificulta a avaliação da adequação das práticas de programação nos fornecedores e sugere a necessidade de investigar os impactos desta multiplicidade de soluções na integração dos sistemas operacionais entre empresas clientes e fornecedoras.

Corrêa e Gianesi (1996) salientam que, para o bom desempenho de um sistema de PCP, a acurácia deve ser superior a $98 \%$. A maioria dos fornecedores pesquisados não alcançou este índice, sugerindo uma baixa precisão dos registros de estoque. Na prática, este problema é atenuado pela revisão freqüente dos inventários.

\section{Conclusões}

A partir dos resultados apresentados na seção anterior, procedeu-se à análise das questões de pesquisa formuladas, as quais dizem respeito à integração dos fornecedores do nível 2 na cadeia automotiva e à adequação das práticas de PCP destes fornecedores.

O desempenho do PCP dos fornecedores foi avaliado conforme as respostas às perguntas sobre pontualidade de entrega e nível de estoque. Em ambos os quesitos (pontualidade de entrega e níveis de estoque), os dados não permitem afirmar que há diferenças significativas entre as camadas, ou seja, o desempenho declarado pelos profissionais de PCP é muito similar nos dois níveis.

Constatou-se também que o grau de colaboração entre clientes e fornecedores é praticamente igual e abaixo do desejado nos dois níveis. Tanto fornecedores do nível 1 quanto do nível 2 declararam que o principal problema para o PCP é a alteração constante e repentina dos pedidos, o que permite concluir que a integração entre clientes e fornecedores na cadeia ainda é insuficiente.

Destaca-se que, no questionário, foi sugerida a classificação por nível de fornecimento. Assumiu-se implicitamente que seria possível classificar os fornecedores exclusivamente por nível e que as respostas seriam uniformes em cada nível. No entanto, o levantamento de dados mostrou que as empresas de uma mesma camada apresentam certa heterogeneidade quanto às práticas de PCP. Esta constatação indica que a estratificação dos fornecedores apenas pela posição na cadeia (nível 1 ou 2) não permite qualificar o grau de coordenação e desempenho, sendo que outros fatores devem ser considerados.

Quanto à adequação das práticas de PCP nos fornecedores, pode-se inferir que há espaço para melhoria significativa destas atividades, conforme os resultados encontrados. Além disso, observou-se que as ferramentas utilizadas são semelhantes em ambos os níveis e que, portanto, os pontos de melhoria aplicam-se também ao nível 1.

Conforme apresentadas na Tabela 2, as ações de melhoria devem concentrar-se nas atividades de programação da produção e previsão de demanda. Quanto à programação da produção, a adoção de modelos de programação com capacidade finita e mecanismos de produção puxada deve proporcionar melhor desempenho aos fornecedores no cumprimento de prazos e na redução de estoques, conforme apontado na literatura (STEVENSON et al., 2005). Um aprimoramento das técnicas de previsão empregadas contribuiria para melhorar a administração de materiais e o planejamento da capacidade de produção. 


\title{
Manufacturing planning and control practices: a survey research on the Brazilian automotive supply chain
}

\begin{abstract}
Automotive suppliers are part of a constantly changing business environment that is subject to oscillations in the economy. Automobile assemblers, the major influence over the rest of the supply chain, have adopted some Japanese management practices (JIT, lean production etc) in order to achieve higher efficiency and lower inventories. This work aims to identify how suppliers along the chain have adjusted to this new situation concerning, mainly, production and inventory planning as well as control. A research was conducted showing that, nonuniformly, suppliers fulfill demand requests satisfactorily in spite of the poor coordination of their nearest clients, low inventory accuracy, and high stock level of raw materials and finished goods. It was also detected that there are no significant performance differences between first and second tier suppliers. It is particularly noteworthy to highlight a special need for production scheduling tools and the huge gap between theory and practice.
\end{abstract}

Keywords: Automotive supply chain. Manufacturing planning and control. Inventory management.

\section{Referências bibliográficas}

ANFAVEA - Associação Nacional dos Fabricantes de Veículos Automotores. Anuário Estatístico. Disponível em: <http:// www.anfavea.com.br>. Acesso em: 30 ago 2007.

ARKADER, R. The perspective of suppliers on lean supply in a developing country context. Integrated Manufacturing Systems, v.12, n.2, p.87-93, 2001.

BITRAN, G.R.; HAAS, E.A.; HAX, A.C. Hierarchical Production Planning: a single stage systems. Operations Research, v.29, n. 4, p.717-743, 1981.

. Hierarchical Production Planning: a two stage systems. Operations Research, v.30, n.2, p.232-251, 1982.

BLACKSTONE, J.H. Theory of constraints: a status report. International Journal of Production Research, v.39, n.6, p.1053-1080, 2001.

BRYMAN, A. Research Methods and Organization Studies. Londres: Unwin Hyman, 1989.

BUXEY, G. Aggregate planning for seasonal demand: reconciling theory with practice. International Journal of Operations \& Production Management, v.25, n.11, p.1083-1100, 2006.

CAGLIANO, R.; CANIATO, F.; SPINA, G. The Linkage between supply chain and manufacturing improvement programmes. International Journal of Operations \& Production Management, v.26, n.3, p.282-299, 2006.

CASTRO, R.L. Planejamento e Controle da Produção e Estoques: um survey com fornecedores da cadeia automotiva brasileira. 2005. 109 f. Dissertação (Mestrado em Engenharia de Produção) - Escola Politécnica, Universidade de São Paulo. São Paulo, 2005. (Disponível em: http://www.teses.usp.br).

CONCEIÇÃO, J.J. As Fábricas do ABC no Olho do Furacão: a indústria de autopeças e a reestruturação da cadeia automotiva nos anos 90. 2001. 224 f. Dissertação (Mestrado) - Centro Universitário Municipal de São Caetano do Sul, São Caetano do Sul, 2001.
CORRÊA, H.L.; GIANESI, I.G.N. Just in Time, MRP II e OPT: um enfoque estratégico. 2.ed., São Paulo: Editora Atlas, 1996.

CHOPRA, S.; MEINDEL, P. Supply Chain Management: strategy, planning and operation. New Jersey: Prentice Hall, 2001.

CSCMP - COUNCIL OF SUPPLY CHAIN MANAGEMENT PROFESSIONALS. Apresenta definições e conceitos sobre gestão de operações e logística. Disponível em: <http://www. cscmp.org >. Acesso em: 01 de junho de 2006.

FORZA, C. Survey research in operations management: a processbased perspective. International Journal of Operations \& Production Management, v.22, n.2, p.152-194, 2002.

FRAMINAN, J.M.; GONZÁLEZ, P.L.; RUIZ-USANO, R. The CONWIP production control system: review and research issues. Production Planning \& Control, v.14, n.3, p.255-265, 2003.

FROHLICH, M.T.; WESTBROOK, R. Arcs of integration: an international study of supply chain strategies. Journal of Operations Management, v.19, n.2, p.185-200, 2001.

FYNES, B.; VOSS, C.; de BÚRCA, S. The impact of supply chain relationship dynamics on manufacturing performance. International Journal of Operations \& Production Management, v.25, n.1, p.6-19, 2005.

GUPTA, J.N.D. An Excursion in Scheduling Theory: an overview of scheduling research in the twentieth century. Production Planning \& Control, v.13, n.2, p.105-116, 2002.

HAX, A.C.; CANDEA, D. Production and Inventory Management, Englewood Cliffs: Prentice-Hall, 1984.

HAX, A.C.; MEAL, H.C. Hierarchical integration of production planning and scheduling. In: M.A. GEISLER (ed.), Studies in Management Science, Amsterdam: North Holland, v.1, p.53-69, 1975.

HINES, P.; HOLWEG, M.; RICH, N. Learning to evolve: a review of contemporary lean thinking. International Journal of 
Operations \& Production Management, v.24, n.10, p.9941011, 2004.

HOPP,W.J.; SPEARMAN, M.L Factory Physics: foundations of manufacturing management. 2.ed., Chicago: Irwin/McGraw Hill, 2000.

HOPP,W.J.; SPEARMAN, M.L. To pull or not to pull: what is the question? Manufacturing and Service Operations Management, v.6, n.2, p.133-148, 2004.

HUANG, G.Q.; LAU, J.S.K.; MAK, K.L. The impacts of sharing production information on supply chain dynamics: a review of the literature. International Journal of Production Research, v.41, n.7, p.1483-1517, 2003.

KARMARKAR, U. Getting control of just-in-time. Harvard Business Review, v. 67, p.122-131, 1989.

LAMBERT, D.M.; COOPER, M.C. Issues in Supply Chain Management. Industrial Marketing Management, v.29, p.65-83, 2000.

LAURINDO, F.J.B.; MESQUITA, M.A. Material Requirements Planning. Gestão \& Produção, v.7, n.3, p.320-337, 2000.

LEE, H.C.; PADMANABHAN, V.; WHANG, S. Comments on "Information Distortion in a Supply Chain: the bullwhip effect". Management Science, v.50, n.12, p.1887-1893, 2004.

MESQUITA, M.A.; SANTORO, M.C. Análise de Modelos e Práticas de Planejamento e Controle da Produção na Indústria Farmacêutica. Revista Produção, v.14, n.1, p.64-77, 2004.

MIRANDA, N.G.M. O Sistema de Avaliação de Desempenho na Cadeia de Suprimentos da Indústria Automobilística Brasileira. 2000, 196 f. Tese (Doutorado) - Escola Politécnica, Universidade de São Paulo. São Paulo, 2000.

OHNO, T. Toyota Production System: beyond large-scale production. Portland: Productivity Press, 1988.

OLHAGER, J.; SELLDIN, E. Supply chain management survey of Swedish manufacturing firms. International Journal of Production Economics, v.89, n.3, p.353-361, 2004.

PLOSSL, G. Orlicky's Material Requirements Planning. 2.ed., Nova York: McGraw Hill, 1994.

POSTHUMA,A.C. Técnicas japonesas de organização nas empresas de autopeças no Brasil. In: CASTRO, N.A. A Máquina e o Equilibrista: inovações na indústria automobilística brasileira. São Paulo: Paz e Terra, p.301-332, 1995.

QUAYLE, M. A study of supply chain management practice in UK industrial SMEs. Supply Chain Management, v.8, n.1, p.7-86, 2003.

ROLDAN, F; MIYAKE, D. A Cadeia de Suprimentos Enxuta: explorando indícios na indústria automobilística brasileira. In: SIMPÓSIO DE ENGENHARIA DE PRODUÇÃO, 10. Bauru, 2003. Anais.... Bauru: Faculdade de Engenharia de Bauru UNESP, 2003.
SALERNO, M.S.; MARX, R.; ZILBOVICIUS,M. A Nova Configuração da Cadeia de Fornecimento na Indústria Automobilística no Brasil. Revista de Administração da USP, v.38. n.3, p.192-204, 2003.

SCHONBERGER, R.J. Applications of single-card and dual-card kanban. Interfaces, v.13, p.56-67, 1983.

SINDIPEÇAS - Sindicato da Indústria de Componentes para Veículos Automotores. Desempenho do Setor de Autopeças. Anuário. São Paulo: Sindipeças, 2005.

SPEARMAN, M.L.; WOODRUFF, D.L.; HOPP, W.J. CONWIP: a pull alternative to kanbam. International Journal of Production Research, v.28, n.5, p.879-894, 1989.

SPENCER, M.S.; COX, J.F. Optimum production technology (OPT) and the Theory of constraints (TOC): analysis and genealogy. International Journal of Production Research, v.33, n.6, p.1495-1504, 1995.

STEELE, D.C.; PHILIPOOM, P.R.; MALHOTRA, M.K.; FRY, T.D. Comparisons between drum-buffer-rope and material requirements planning: a case study. International Journal of Production Research, v.43, n.15, p.3181-3208, 2005.

STEVENSON, M.; HENDRY, L.C.; KINGSMAN, B.G. A review of production planning and control: the applicability of key concepts to the make-to-order industry. International Journal of Production Research, v.43, n.5, p.869-898, 2005.

STOOP, P.P.M.; WIERS, V.C.S. The complexity of scheduling in practice. International Journal of Operations \& Production Management, v.16, n.10, p.37-53, 1996.

SZWEJCZEWSKI, M.; LEMKE, F.; GOFFIN, K. Manufacturersupplier relationships: an empirical study of German manufacturing companies. International Journal of Operations \& Production Management, v.25, n.9, p.875-897, 2005.

VOLLMANN, T.E. BERRY, W.L.; WHYBARK, D.C. Manufacturing Planning and Control Systems. 4.ed. Nova York: McGraw-Hill, 1997.

WOMACK, J.P.; JONES, D.T. Lean Thinking: banish waste and create wealth in your corporation. Nova York: Simon \& Schuster, 1996.

WOMACK, J.P.; JONES, D.T.; ROOS, D. A Máquina que Mudou o Mundo. Rio de Janeiro: Campus, 1992.

WU, Y.C. Lean manufacturing: a perspective of lean suppliers. International Journal of Operations \& Production Management, v.23, n.11, p.1349-1376, 2003.

\section{Sobre os autores}

\section{Marco Aurélio de Mesquita \\ Roberto Lopes de Castro}

Departamento de Engenharia de Produção, Escola Politécnica, Universidade de São Paulo, Av. Prof. Almeida Prado, $n^{\circ}$ 531, CEP 05508-070, São Paulo, SP, Brasil,

e-mails: marco.mesquita@ poli.usp.br; rldecastro@bol.com.br 